\title{
PENSAMIENTOS DE PRIMER ORDEN
}

Mariela Aguilera

Instituto de Humanidades

Universidad Nacional de Córdoba

CONICET

maguilera@psyche.unc.edu.ar

RESUMEN: Uno de los argumentos en favor de la dependencia entre lenguaje y conceptos descansa en la premisa de que la posesión de conceptos involucra pensamientos de segundo orden y éstos, a su vez, requieren lenguaje. Este trabajo se centra en una variante de este argumento formulada por José Luis Bermúdez. Sostendré que aun cuando el pensamiento de segundo orden suponga competencia lingüística, no es necesario aceptar esa premisa. Propondré, en cambio, dos condiciones alternativas para la posesión de conceptos, la identificación y la generalidad. Si bien ninguna de ellas conlleva el pensamiento de segundo orden, en forma conjunta reflejan importantes aspectos del pensamiento conceptual.

PALABRAS CLAVE: conceptos, pensamiento de segundo orden, inferencias, requisito de generalidad, identificación

SUMMARY: One argument for the interdependence of language and concepts relies on the assumption that concept possession involves second order thoughts, which in turn are language dependent. This paper focuses on a recent version of this argument formulated by José Luis Bermúdez. It claims that even though second order thought involves linguistic competence, the above assumption is not compelling. Instead, two different conditions for concept possession are proposed, identification and generality. Although none of them involves second order thought, together they highlight important features of conceptual thinking.

KEY WORDS: concepts, second order thought, inferences, generality constraint, identification

Knowing that I am thinking of Alice is surely posterior rather than prior to thinking of Alice. I cannot know that I am thinking of Alice unless I first think of Alice, any more than I can know that I am hungry unless I am first hungry. Nor is knowing that I am thinking of Alice necessitated by my thinking of Alice. Knowing that requires judging that, and judging that I am thinking of Alice requires the capacity to think about thoughts. But this is a capacity there is no reason to suppose every thinker must have.

(Millikan 2000, p. 179) 


\section{Introducción}

Muchos filósofos han defendido la existencia de una dependencia entre lenguaje y conceptos a lo largo de la historia. ${ }^{l}$ En su favor se han formulado diversos tipos de argumentos. En este trabajo, me centraré en una de las tesis en las cuales se ha apoyado un subgrupo de esos argumentos, a saber, la tesis de que (i) para tener pensamiento conceptual, una criatura debe ser capaz de pensar en sus propios pensamientos, por lo cual debe tener pensamientos de segundo orden. ${ }^{2}$ En particular, me enfocaré en una versión reciente de esta tesis, que Bermúdez propone en diversos lugares. Según él, (T) el pensamiento de segundo orden (o ascenso intencional) requiere pensar acerca de nuestras palabras (o ascenso semántico). En su favor, Bermúdez sostiene que (ii) los conceptos se individúan por su papel inferencial y (iii) sólo a través del lenguaje natural una criatura puede comprender las condiciones de individuación de los conceptos, pues (ii.i) el papel inferencial del pensamiento depende de sus rasgos estructurales y (ii.ii) sólo un lenguaje posee una estructura canónica capaz de reflejar las propiedades estructurales del pensamiento.

Contra ese argumento podrían citarse numerosas investigaciones en psicología comparada que sugieren que algunos primates no humanos, carentes de lenguaje, son capaces de realizar lo que los filósofos han llamado lectura de mente; esto es, pueden atribuir estados mentales a otros individuos (Call y Tomasello 2008, Call et al. 2004, Hare et al. 2000). Si la lectura de mente requiere la capacidad de representar otros pensamientos, estas investigaciones cuestionarían la

${ }^{1}$ Dentro de la tradición analítica, muchos autores consideran que hay una dependencia entre conceptos y lenguaje natural (Bermúdez 1998, 2010, Brandom 2000, Davidson 1982, Hurley 2003). Los autores de orientación psicológica consideran, en cambio, que el pensamiento conceptual involucra un lenguaje del pensamiento (Fodor 2008, Fodor y Pylyshyn 1988). Si bien ambos grupos comparten el supuesto de que la posesión de conceptos requiere vehículos lingüiformes, el argumento que me interesa criticar aquí se aplica sólo al primer grupo.

${ }^{2}$ Tradicionalmente se ha vinculado la noción de pensamiento reflexivo con la de pensamiento de segundo orden. Si, como se acepta comúnmente, un pensamiento de segundo orden es un pensamiento acerca de otro pensamiento y si el pensamiento conceptual conlleva pensamiento de segundo orden, entonces, tener conceptos estaría vinculado con otras capacidades metarrepresentacionales, como la evaluación y la justificación epistémica (Davidson 1982, McDowell 1994) y la lectura de mente (Bermúdez 2010). A menos que lo indique el contexto, me atendré a este uso tradicional. Sin embargo, algunos autores desvinculan la capacidad de reflexionar del pensamiento de segundo orden (Lurz 2009), y otros distinguen la metarrepresentación de la metacognición, la primera de las cuales se encuentra asociada con el pensamiento de segundo orden, mientras que la segunda no (Proust 2006). Volveré sobre la noción de Bermúdez de pensamiento reflexivo en la sección 2. 
tesis de que el pensamiento de segundo orden requiere competencia lingüística. Pero el argumento de Bermúdez parece impermeable a este tipo de crítica, pues parece rechazar a priori que sin lenguaje se pueda pensar acerca de otros pensamientos. En consecuencia, quien quiera defender que los animales tienen la capacidad de leer otras mentes tendrá que dirigirse a este último argumento pues la presentación de evidencia empírica no parece suficiente para mostrar que los animales pueden tener pensamientos de segundo orden. ${ }^{3}$ No me detendré, sin embargo, en la estructura completa de este argumento, ya que resulta mucho menos claro que debamos aceptar la premisa, sobre la cual se asienta, de que el pensamiento de segundo orden es un requisito para poseer conceptos. En otras palabras, aun cuando aceptemos que el ascenso intencional involucra ascenso semántico, no creo que debamos aceptar el mismo punto de partida que adopta Bermúdez (la premisa i). De hecho, el mismo Bermúdez parece admitir que el pensamiento de segundo orden no es inherente a todo tipo de pensamiento conceptual y sugiere restringir el requisito a sus manifestaciones más elevadas. Pero, como sostendré aquí, esta interpretación no es viable para Bermúdez.

Este trabajo tiene dos objetivos: uno de ellos interpretativo, el otro crítico. En la sección 2, mostraré que entre las motivaciones para aceptar el requisito de ascenso intencional se encuentra el compromiso de Bermúdez con una concepción internista del papel inferencial, que lleva implícita la exigencia de que la posesión de conceptos se asienta en capacidades reflexivas. A continuación, sostendré contra Bermúdez que la capacidad de hacer inferencias no involucra necesariamente la capacidad de representar explícitamente las cadenas inferenciales, tal como supone el internismo. Para concluir la sección 2, sostendré que aun cuando admitamos que por medio del requisito de ascenso intencional se obtiene una distinción tajante entre las criaturas que tienen conceptos y aquellas que no, la apelación a este requisito no ilumina de manera significativa para qué sirven

${ }^{3}$ Aun cuando ésta constituye una interesante vía argumentativa contra el vínculo entre el pensamiento de segundo orden y el lenguaje natural, no es la que desarrollaré aquí. Nótese que el hecho de que la evidencia empírica sobre la lectura de mente no resulta suficiente para refutar el argumento de Bermúdez que vincula la lectura de mente con la competencia lingüística, no significa que sea irrelevante e inocua al momento de ponderar ese argumento. Lo que quiero enfatizar es que debe estar respaldada por desarrollos conceptuales, pues es posible que los estudios empíricos muestren que los animales tienen capacidades para la cognición social más básicas que las que exige Bermúdez para hablar de una genuina lectura de mente. 
los conceptos y excluye buena parte de lo que podría considerarse pensamiento conceptual.

En contraposición, en la sección 3, sostendré que lo que distingue a las criaturas que poseen conceptos de las que no los tienen es la satisfacción de dos condiciones diferentes a la condición que propone Bermúdez: por un lado, la identificación y, por el otro, la generalidad. Ninguna de estas dos condiciones, por su parte, involucra ascenso intencional. De mi argumento se sigue que el requisito de ascenso intencional excluye precisamente los pensamientos conceptuales que, sin ser reflexivos, permiten identificar y hacer inferencias, y satisfacen el requisito de generalidad. Y, lo que es más importante, ambas condiciones en forma conjunta expresan de un modo iluminador importantes aspectos del pensamiento conceptual y, en particular, ponen de manifiesto el papel cognitivo que comporta la realización de inferencias para una criatura pensante.

\section{El carácter reflexivo del pensamiento conceptual}

"Two Arguments for the Language-Dependence of Conceptual Thought" (2010) se suma a los diversos intentos de Bermúdez por defender la dependencia entre los conceptos y el lenguaje natural (Bermúdez 1998, 1995). En ese artículo Bermúdez apela a una línea argumentativa hasta entonces inconexa, y defiende que un tipo especial de pensamiento, el pensamiento de segundo orden, requiere competencia lingüística. ${ }^{4}$ El autor explícitamente ha propuesto desvincular esta línea argumentativa de su propuesta general sobre el pensamiento conceptual (Bermúdez 2003), ya que resulta mucho menos convincente que toda forma de pensamiento conceptual requiera lenguaje. Sin embargo, en su reciente artículo (2010) convergen el argumento, más general, sobre el carácter lingüístico del pensamiento conceptual y el argumento, más específico, sobre la naturaleza lingüística del pensamiento de segundo orden. El nexo para esta vinculación radica en el supuesto, defendido en The Paradox of Self

\footnotetext{
${ }^{4}$ Esta línea argumentativa se ha desarrollado en sus trabajos sobre la dinámica cognitiva de segundo orden, que incluye la lectura de mente, la evaluación de razonamientos, la revisión de deseos, etc. (Bermúdez 2009, 2003). En estos trabajos la exigencia de un lenguaje natural se restringe al pensamiento acerca de otros pensamientos (y no al pensamiento en general). En "Mindreading in the Animal Kingdom" (2009), Bermúdez sostiene que sólo un lenguaje natural —en contraposición con un lenguaje del pensamiento - puede desempeñar este papel ya que las representaciones explotadas en la lectura de mente — práctica que parece requerir el pensamiento de segundo orden - deben ser conscientes o de nivel personal, para que se puedan usar en la toma de decisiones prácticas de un agente.
} 
Consciousness (1998), acerca del carácter reflexivo del pensamiento conceptual. Es posible decir que de esta manera se ve reforzado el vínculo entre conceptos y lenguaje natural, puesto que se clarifica por qué el carácter reflexivo del pensamiento conceptual requeriría competencia lingüística.

De esta manera, la propuesta de Bermúdez se sitúa junto a la de autores como Davidson (1982), Brandom (2010) y McDowell (1994), entre los más destacados, que consideran que la reflexividad es el rasgo esencial del pensamiento conceptual. ${ }^{5}$ Las razones para defender esta tesis están fundadas - a mi entender- en la concepción general sobre los conceptos que presupone cada uno de estos autores. Para McDowell, el carácter reflexivo del pensamiento conceptual da cuenta de la actividad cognitiva que involucra el uso de conceptos. En el caso de Davidson y Brandom podría decirse que el carácter reflexivo del pensamiento conceptual se encuentra estrechamente vinculado con su caracterización del carácter normativo de los conceptos. De este modo, Davidson (1982) sostiene que, para tener conceptos, una criatura debe ser capaz de reconocer sus errores; sólo así se puede dar cuenta del contraste entre lo objetivo y lo subjetivo, entre la verdad y la falsedad inherentes al pensamiento. De manera similar, Brandom (2010) sostiene que sin llevar la cuenta discursiva, es decir, sin volverse reflexivamente sobre tales prácticas, los contenidos proposicionales no podrían tener condiciones de verdad objetivas.

Ahora bien, ¿qué motivaciones han llevado a Bermúdez a adoptar este requisito? No resulta fácil responder esta pregunta, pues él mismo parece no estar preocupado por responderla. En el caso de la lectura de mente resulta más claro por qué se vería involucrado el ascenso intencional, ya que esta práctica se define como la capacidad que tiene una criatura de pensar en el pensamiento de sus congéneres (Bermúdez 2009). Pero la relación entre tener conceptos y tener pensamientos de segundo orden, en cambio, no resulta tan evidente. En su artículo de 2010 (p. 44), Bermúdez incluso sugiere que quienes no estén convencidos de que el pensamiento de segundo orden sea la marca distintiva del pensamiento conceptual podrían relativizar su argumento y aceptar, al menos, que su criterio permite distinguir las manifestaciones más elevadas del pensamiento conceptual (como la revisión explícita y deliberada de creencias y deseos, por ejemplo).

\footnotetext{
${ }^{5}$ La adopción de este requisito permite distinguir este grupo de neofregeanos de un segundo grupo que considera que es el requisito de generalidad el que marca la diferencia entre el contenido conceptual y el no conceptual (Evans 1982, Peacocke 1992, Heck 2007). Volveré sobre este requisito más adelante.
} 
De esta manera, la adopción generalizada de este requisito podría considerarse, por lo menos, arbitraria si lo que nos interesa es la posesión de conceptos per se y no sólo sus manifestaciones más elevadas. Sin embargo, Bermúdez sostiene que su caracterización delimita una importante clase natural epistémica, sin importar si refleja o no el uso filosófico habitual de "concepto", ni qué otro tipo de etiqueta le adosemos (Bermúdez 2010, p. 44). Pero entonces habría que ver si esta caracterización resulta lo suficientemente inclusiva e interesante como para iluminar el papel cognitivo de los conceptos o bien excluye buena parte de nuestros pensamientos conceptuales.

No creo, sin embargo, que la posición de Bermúdez se pueda relativizar del modo en que él propone. Sostendré que el requisito de reflexividad se encuentra fuertemente arraigado en la propia concepción internista del papel inferencial que defiende este autor en The Paradox of Self Consciousness (Bermúdez 1998). Como mostraré a continuación, la aceptación de la posibilidad de tener conceptos sin tener al mismo tiempo un conocimiento explícito de sus condiciones de individuación no es compatible con tal concepción. El argumento que propone Bermúdez, por otra parte, lo compromete a aceptar que el conocimiento práctico o implícito de esas condiciones depende, en última instancia, de su conocimiento explícito. ${ }^{6}$ De este modo, la noción de concepto excluye pensamientos que satisfacen otras condiciones significativas para el pensamiento conceptual pero que no involucran una comprensión explícita de sus condiciones de individuación. Por esta razón constituye una noción demasiado restrictiva.

Dentro de la propuesta de Bermúdez, el carácter reflexivo de los conceptos debe entenderse en los siguientes términos: las criaturas con pensamiento conceptual han de tener una comprensión reflexiva de: a) las relaciones lógicas entre los pensamientos, b) las relaciones de evidencia entre percepciones, creencias y pensamientos, y c) las relaciones de justificación entre pensamientos y acciones (Bermúdez 2010, p. 44). Mi hipótesis es que esta exigencia, así definida, depende de su concepción inferencial e internista de los conceptos, según la cual i) los conceptos se individúan —al menos parcialmente- en

\footnotetext{
${ }^{6}$ Puede ser conveniente hacer una aclaración terminológica. De acuerdo con Bermúdez (1998), implícito aquí significa no consciente de manera explícita o no reflexivo. Este tipo de comprensión podría presuponerse, por ejemplo, cuando se usa un concepto sin reparar de manera deliberada o reflexiva en sus condiciones de individuación o cuando una criatura es capaz de hacer las transiciones inferenciales adecuadas, aun sin pensar explícitamente - esto es, de manera consciente - en las reglas que rigen tales transiciones. La representación explícita, por su parte, requiere ascenso intencional.
} 
virtud de su papel inferencial, esto es, en virtud de la contribución al poder inferencial de las proposiciones en las que aparece (Bermúdez 1995) y ii) la maestría en un concepto involucra la comprensión - ya sea implícita o explícita - de sus condiciones de individuación, en particular, de su papel inferencial (1998, p. 67). Cabe aclarar que la tesis acerca del carácter reflexivo del pensamiento conceptual, en sentido estricto, no es presupuesta tanto por (ii), la condición para la posesión de conceptos, sino que ya se ve implicada en (i), la noción de conceptos que el autor adopta, pues la concepción inferencialista que adopta Bermúdez se encuentra comprometida con una concepción internista de la justificación.

Mi tesis es que no se puede renunciar a la tesis sobre el carácter reflexivo del pensamiento conceptual y mantener, al mismo tiempo, una concepción internista del papel inferencial, como la que defiende explícitamente Bermúdez (1998). ${ }^{7}$ Dicho de otro modo, si Bermúdez quisiera abandonar la tesis sobre el carácter reflexivo de los conceptos debería renunciar también a gran parte de la propuesta sobre los conceptos que defiende en The Paradox of Self Consciousness (1998). Por medio de tal caracterización de la noción de concepto y de sus condiciones de posesión (i y ii), Bermúdez sostiene que, para tener un concepto, una criatura no sólo ha de ser sensible a las transiciones inferenciales sino que además tiene que ser capaz de proveer justificaciones de tales transiciones, esto es, de "articular las razones para una clasificación, inferencia o juicio dado" (1998, p. 71). De este modo, la concepción de Bermúdez excluye de lo conceptual los contenidos mentales que se requieren para explicar los experimentos de deshabituación realizados con niños en etapa prelingüística y, de manera semejante, los procesos inferenciales que subyacen a diversos tipos de conducta animal, así como aquellos que se dan en los mecanismos subpersonales. Veamos.

En primer lugar, de acuerdo con una concepción inferencialista, un concepto se individúa conforme a diversas relaciones inferenciales que comparte con otros conceptos. Por ejemplo, el pensamiento de que Fonta es un perro se relaciona inferencialmente con el pensamiento de que Fonta es un animal en virtud de las relaciones inferenciales entre el concepto de PERRO y el concepto de ANIMAL. En este sentido, cualquier concepto se encuentra anclado en una red

${ }^{7}$ Por concepción internista del papel inferencial me estoy refiriendo a la conjunción entre inferencialismo respecto de los conceptos e internismo respecto de la justificación. No obstante, es cierto que de esta conjunción se deriva, a continuación, un internismo respecto de los conceptos. 
de conceptos más amplia y la aplicación de un concepto presupone la capacidad para llevar a cabo un buen número de inferencias donde el concepto aparece. Así pues, la aplicación del concepto PERRO a Fonta supone, por ejemplo, la creencia de que los perros son animales y se conecta, por lo tanto, con el pensamiento de que Fonta es un ANIMAL así como con otros pensamientos acerca del eventual comportamiento de Fonta (que, como tal, tiene movimiento propio, es amigable, puede enojarse y morder, etc.). Más aún, si las condiciones de individuación de los conceptos se identifican con el papel inferencial que cumplen en los pensamientos, ha de haber un vínculo conceptual entre tener conceptos y tener actitudes proposicionales, en el sentido de que la aplicación de un concepto $P$ a un objeto $x$ involucra una actitud doxástica hacia la proposición $x$ es $P$ (aunque no a la inversa). ${ }^{8}$

En segundo lugar, Bermúdez afirma que la aplicación de un concepto es inseparable de la realización de juicios clasificatorios. En este tipo de juicios, un objeto se subsume en una categoría conforme a un conjunto de razones. En consecuencia, la posesión de un concepto no sólo implica la realización de un buen número de inferencias que se siguen de su aplicación sino también presupone que sus aplicaciones están justificadas, esto es, el pensamiento en el que aparece un concepto dado puede funcionar o bien como premisa o bien como consecuencia de otras proposiciones (Brandom 2000).

En tercer lugar, Bermúdez distingue la mera sensibilidad a las transiciones inferenciales de la sensibilidad racional a ellas. La sensibilidad racional involucra la capacidad para justificar las transiciones lógicas y evidenciales de un pensamiento a otro, pero ser simplemente sensible a las transiciones inferenciales no. Para reforzar este contraste, Bermúdez (1998, p. 71) se apoya en la distinción tomada de la epistemología entre estar justificado para sostener una creencia y justificar esa creencia: podría haber razones o causas externas para creer que $p$, de modo tal que $\mathbf{S}$ está justificado para creer que $p$ sin ser capaz de reconocer las razones ni, mucho menos, de justificar su creencia de que $p .{ }^{9}$ Esto es todo lo que presupone la noción de

${ }^{8}$ Para Bermúdez (1998), el contenido de las actitudes proposicionales puede ser no conceptual y, por lo tanto, tener actitudes proposicionales no necesariamente entraña la capacidad para realizar juicios.

${ }^{9}$ Contra muchos filósofos de su misma tradición, Bermúdez (2003) sostiene que los animales sin lenguaje pueden tener actitudes proposicionales, como creencias y deseos. De acuerdo con esto, se puede afirmar que un mono, por ejemplo, podría estar justificado para creer que está en peligro tras haber escuchado el grito de alarma de uno de sus congéneres aun cuando él mismo sea incapaz de justificar 
justificación externista. Para una concepción internista de la justificación, en cambio, "sólo las creencias u otros estados internos al sujeto sirven para determinar cuándo está justificada una creencia" (Eraña 2009, p. 47). Este desplazamiento del ámbito de las inferencias al de las creencias adquiere mayor respaldo si aceptamos - al igual que Bermúdez - la estrecha conexión entre conceptos, juicios y creencias, de modo que la justificación de creencias se vuelve un aspecto relevante para entender la noción de conceptos, tal como la caracteriza este autor. ${ }^{10}$

Por último, Bermúdez sostiene que la sensibilidad racional es una condición necesaria para tener conceptos. Por este motivo, no basta que una criatura tenga justificación para hacer ciertas transiciones inferenciales y decir que está aplicando un concepto. En otras palabras, las razones no sólo deben respaldar los juicios clasificatorios de la criatura sino que también deben ser accesibles a ella, es decir, deben poder ser reconocidas y usadas para fines epistémicos. A esto, Bermúdez agrega la exigencia de comprender las reglas generales de inferencias y reconocer que su aplicación es apropiada en una situación dada (1998, p. 70). ${ }^{11}$

Como esta reconstrucción deja ver, la noción de conceptos a la que Bermúdez se adscribe se encuentra articulada con una concepción internista de la justificación. ${ }^{12}$ De acuerdo con esta concepción, un juicio debe ser respaldado por razones internas a las que la criatura

activamente su creencia ni la conducta motivada por la amenaza en cuestión. Pueden encontrarse innumerables ejemplos de este tipo en Cheney y Seyfarth 2007 y 1990. Los chimpancés, por su parte, son capaces de inferir ciertas propiedades funcionales de algunos objetos, tal como se manifiesta en la modificación y el uso de objetos como herramientas (Boesch y Boesch, 1990). No obstante, aunque ello dé cuenta de que estos simios son capaces de razonar (Glock 2009, 2000), no muestra en lo absoluto que puedan dar justificaciones, que es una práctica paradigmáticamente lingüística.

${ }^{10} \mathrm{Si}$ la aplicación de un concepto se vincula íntimamente con la realización de un juicio, es natural aceptar la existencia de una íntima relación entre conceptos y creencias. Ésta se sigue del siguiente hecho: el juicio, realizado por un sujeto $\mathrm{S}$, "Creo que $x$ es $P$ " se vuelve redundante en los contextos usuales, pues el juicio " $x$ es $P$ " ya expresa la creencia de que " $x$ es $P$ ".

${ }^{11}$ Esta última condición, en la que no puedo centrarme por completo aquí, muestra cuán exigente e inadecuada resulta la noción de concepto que defiende Bermúdez, en tanto que vuelve la posesión de conceptos muy dificultosa incluso respecto de los humanos lingüísticamente competentes.

${ }^{12}$ No es mi intención centrarme en la disputa internismo-externismo respecto de la noción de justificación. He traído a colación tal distinción con el único fin de esclarecer los supuestos que llevan a Bermúdez a defender el vínculo entre pensamiento de segundo orden y conceptos. Si bien mi crítica se centrará en las dificultades que surgen a partir de ese vínculo, si se toman en cuenta las habilidades lógicas 
pensante puede acceder a partir de la reflexión, esto es, razones de las cuales ha de ser consciente: "La idea de fondo es que los factores que determinan la justificación de una creencia son condiciones a las cuales el sujeto (S) tiene (o puede tener) acceso cognitivo directo" (Eraña 2009, p. 49).

En consecuencia, el carácter reflexivo del pensamiento conceptual es inherente a la concepción de los conceptos que Bermúdez propone, pues si los conceptos se constituyen por su papel inferencial y, como él sostiene, "los casos paradigmáticos de inferencia son actos reflexivos y conscientes" (1998, p. 70), la posesión de conceptos necesariamente ha de suponer capacidades que pueden volverse reflexivas y conscientes. En consecuencia, si aceptamos la concepción de Bermúdez, el requisito de ascenso intencional no puede ser relativizado a las formas más elevadas de conceptualización sino que, por el contrario, deberíamos aceptar que el pensamiento de segundo orden es necesario para tener cualquier tipo de conceptos.

Es cierto que Bermúdez admite que el uso habitual de conceptos sólo requiere un conocimiento práctico e implícito de sus condiciones de individuación. Sin embargo, la noción de justificación a la que el autor se adscribe implica que para tener conceptos una criatura pensante debe ser consciente, al menos en algunos casos, de las relaciones lógicas y evidenciales en las que éstos participan. En otras palabras, esto implica que el conocimiento práctico e implícito de las relaciones inferenciales que se requiere para el uso de conceptos depende, en última instancia, de la capacidad para representar explícitamente tales relaciones.

Contra esa tesis podríamos alegar que los niños comienzan a usar conceptos léxicos mucho antes de ser capaces de representar explícitamente sus relaciones inferenciales. Pero a esto Bermúdez puede responder que hasta antes de alcanzar la facultad de reflexionar no habrían adquirido conceptos. Esta respuesta pierde su fuerza si se tiene en cuenta que el desarrollo de la capacidad para representar explícitamente las relaciones estructurales entre nuestro pensamiento llega tardíamente, al término de la niñez o en la adolescencia tem-

de niños y animales no lingüísticos, pueden citarse otras objeciones. Por ejemplo, Destéfano (2009) ha argumentado que la introducción de nociones epistémicas como la de justificación dentro de una teoría del contenido resulta problemática, en primer lugar, porque introduce una extensa e irresuelta discusión de la epistemología y, en segundo lugar, porque las cuestiones relativas a los contenidos conceptuales son de orden metafísico, no epistémico. Sin embargo, simpatizo con una concepción de los conceptos como habilidades, según la cual la posesión de conceptos es inseparable de la posesión de algún tipo de conocimiento. 
prana: mucho después de usar un lenguaje, de razonar, de percatarse de la realización de inferencias, de atribuir actitudes proposicionales a sí mismos y a terceros e, incluso, de ser consciente del estatus y alcance de sus estados epistémicos, etc. (Lurz 2007, pp. 277-278). Como señala Lurz, los niños de alrededor de cuatro años que pasan el test de falsa creencia no adquieren sino mucho más tarde la capacidad de reflexionar en forma explícita sobre sus pensamientos. ${ }^{13}$ Por otro lado, apenas a los cuatro años comienzan a percatarse de que parte de lo que saben procede de hacer inferencias (Lurz 2007). Esto pone de manifiesto que no hay una conexión conceptual entre el ascenso intencional y el pensamiento explícito reflexivo, como Bermúdez parece suponer.

En esta misma dirección, Call (2006a) sostiene que muchos simios son sensibles a sus estados epistémicos: reconocen su falta de información, ya sea por olvido o ignorancia. Sin embargo, si hemos de aceptar que los animales pueden reflexionar sobre sus estados epistémicos debemos admitir que lo hacen de modo implícito. Muchos estudios señalan que el razonamiento instrumental es una facultad extendida en diversos primates no humanos (Boesch y Boesch 1990, Cheney y Seyfarth 2007 y 1990; Call 2006a) e incluso en algunas especies de pájaro (Bond et al. 2003, Weir y Kacelnik 2006, Clayton et al. 2006). Incluso el mismo Bermúdez (2003) ha defendido que algunos animales son capaces de hacer inferencias de un modo implícito, o - como él las llama- protoinferencias, esto es, inferencias que preservan el valor de verdad pero no en virtud de su forma. Pero, a menos que aceptemos también que los animales sin lenguaje pueden representar explícitamente las relaciones lógicas y evidenciales entre sus pensamientos, el hecho de que sean capaces de hacer (proto)inferencias e incluso de reflexionar de un modo implícito exige renunciar a la tesis de que el conocimiento implícito de las condiciones de individuación de los conceptos depende de la capacidad para representar explícitamente su papel inferencial.

Cabe aclarar que mi idea no es negar que sea necesario conocer las condiciones de individuación de los conceptos ni tampoco que el papel inferencial forme parte de tales condiciones. Pretendo, en cambio, rechazar que el conocimiento implícito de éstas depende de su conocimiento explícito y, por otra parte, resaltar el papel que

${ }^{13}$ Por el contrario, alrededor de los cinco años los niños son capaces de evaluar epistémicamente y ponderar el estatus de su conocimiento sobre sus propios estados mentales (Mitchell et al. 2009). Ello no implica, sin embargo, que en ese mismo periodo los niños sean conscientes de la estructura lógica de sus pensamientos. Agradezco a uno de los evaluadores anónimos por ponerme al tanto de este artículo. 
cumplen otras condiciones que son, a mi entender, relativamente más básicas y centrales. En el apartado siguiente, ofreceré otro argumento en contra de la dependencia entre lo implícito y lo explícito que, al mismo tiempo, integra el inferencialismo con una visión representacionalista de los conceptos y sus condiciones de posesión. Para ello, propondré dos condiciones diferentes para la posesión de conceptos: la identificación y la generalidad. Mi idea es que la identificación es una habilidad más básica que la de hacer inferencias y es imprescindible para dar cuenta de ella. En particular, la identificación proporciona otro modo de individuar los conceptos cuya comprensión no requiere el conocimiento explícito que requiere Bermúdez. La generalidad, por su parte, constituye un requisito para la posesión de conceptos que no involucra ascenso intencional (o al menos no el tipo de pensamiento explícito en el que piensa Bermúdez). Mi hipótesis es que si una criatura satisface ambas condiciones tendríamos buenas razones para admitir que tiene conceptos. ${ }^{14}$

Estas condiciones, por otra parte, ponen de manifiesto el tipo de pensamiento conceptual que quedaría excluido por medio del requisito de ascenso intencional; precisamente el tipo de pensamiento que, sin ser reflexivo, satisface la identificación y la generalidad. Reconozco que, si bien no se trata de un argumento concluyente, sí es bastante persuasivo. No obstante, quien quiera mantener el ascenso intencional como una condición para tener conceptos, deberá ofrecer razones adicionales para excluir otras variantes de pensamiento. Es importante agregar que mi argumentación no depende de encontrar casos de criaturas que posean conceptos sin ser capaces de tener pensamientos de segundo orden, sino más bien de mostrar que es posible, al menos teóricamente, que una criatura tenga conceptos aun cuando no posea las capacidades reflexivas explícitas que exige Bermúdez.

\footnotetext{
${ }^{14}$ Es importante aclarar que, al adscribirse a estas dos condiciones, se ve garantizado el papel inferencial de los conceptos. Como argumento más adelante, mientras que la identificación es imprescindible para dar cuenta de la realización de inferencias, una criatura no podrá satisfacer el requisito de generalidad a menos que sea capaz de realizar inferencias. En este sentido, mi propuesta debe entenderse como un enfoque mixto sobre los conceptos, según el cual éstos involucran habilidades inferenciales e identificatorias. Por esta razón, mi propuesta recupera elementos tanto de la teoría de Bermúdez como de la de Millikan, para intentar sortear las dificultades de cada una de ellas. En específico, he centrado mis esfuerzos en criticar el aspecto internista del inferencialismo de Bermúdez, que lo lleva a aceptar que el pensamiento de segundo orden y la justificación epistémica son inherentes a la noción de concepto.
} 


\section{Dos marcas de lo conceptual: identificación y generalidad}

La concepción de Bermúdez de los conceptos pierde de vista un importante logro cognitivo resultado de la adquisición de éstos, a saber, la capacidad de reconocer que distintos trozos de información no pertenecen sino a un mismo objeto (Millikan 2000, Duhau 2010). Esta función se denomina comúnmente identificación de objetos o de sustancias (Millikan, 2000). ${ }^{15}$ Esta capacidad, y no la de volvernos epistémicamente sobre nuestro pensamiento, recoge de modo significativo uno de los papeles centrales que cumple la capacidad de realizar inferencias para quien posee conceptos. El problema no es que Bermúdez no pueda ulteriormente dar cuenta de tal función, sino que no la considera dentro de los aspectos centrales de su teoría. Si así lo hiciera, probablemente no consideraría que el carácter explícito y reflexivo del pensamiento sean rasgos constitutivos de los conceptos.

\section{a) Identificación}

Tal como muchos filósofos lo reconocen, la representación es una de las funciones cognitivas básicas de los conceptos.

Cualquier teoría del pensamiento debería empezar con la tarea básica del pensamiento: representar el mundo. Las creencias representan el mundo como si fuera de cierta manera, mientras que los deseos lo representan del modo en que debería ser, a la luz de una criatura pensante $[\ldots]$. Estos criterios básicos para el pensamiento nos brindan una comprensión de las tareas básicas de los conceptos. Donde una creencia o un deseo representa un estado de cosas completo y se especifica típicamente por medio de un enunciado completo, como "Esta manzana es roja", un concepto representa una cosa particular o un modo de ser particular de algo y, por lo tanto, se especifica típicamente sólo por medio de una palabra o frase como "esta manzana" o "es roja". (Camp 2009, p. 276)

Los conceptos refieren a, recogen, denotan o bien tienen una relación intencional con individuos o conjuntos de entidades, eventos, estados, propiedades, etc. (Weiskopf 2009, p. 147)

${ }^{15}$ Millikan (2000) es tal vez la autora que más se ha esforzado por distinguir esta función cognitiva de los conceptos de otras funciones como la clasificación, la discriminación, etc. Otros autores, como Evans (1982), Strawson (1959) y Peacocke (1992), consideraron — al igual que Millikan - que el pensamiento sobre particulares es una forma básica de pensamiento. Sin embargo, a diferencia de ella, asumieron que se trata de una capacidad privativa de las criaturas lingüísticas, que involucra pensamiento con una estructura proposicional al igual que otras capacidades cognitivas. 
Ahora bien, no es la representación por sí misma sino la integración de diversas representaciones sobre un mismo objeto y su uso en diferentes circunstancias lo que dota a una criatura con conceptos de una importante herramienta cognitiva. Más específicamente, la habilidad para identificar objetos cumple un destacado papel en la facilitación y explotación de las diversas interacciones de una criatura pensante con su entorno, pues la identificación permite ampliar el conocimiento acerca de los objetos particulares y, además, es una habilidad necesaria para la realización de inferencias ampliativas. De hecho, un sistema podría ser representacional y, no obstante, carecer de conceptos. Esto es algo que las nociones de representación y contenido no conceptual parecen suponer. Veamos.

Bermúdez admite que, sin lenguaje y sin conceptos, una criatura puede integrar información relevante del ambiente. Es sabido que él es un defensor de los contenidos no conceptuales y de su atribución a criaturas no lingüísticas. ${ }^{16}$ Los contenidos no conceptuales están compuestos por representaciones que no son conceptos; no obstante, al igual que los contenidos conceptuales, tienen una estructura composicional, esto es, pueden descomponerse en elementos que a su vez pueden formar parte de nuevas combinaciones. Al tener una estructura composicional, los contenidos no conceptuales son aptos para la integración cognitiva, esto es, para la interacción entre estados representacionales y motivacionales, así como de estados representacionales entre sí. La integración cognitiva, por su parte, permite usar la información acumulada previamente en nuevas ocasiones.

[P]arte de lo que está involucrado en la integración de estados representacionales es que una criatura que represente el entorno debería ser capaz de registrar cuándo el entorno es relevantemente similar a lo largo del tiempo. El ejemplo más simple sería cuando el entorno que se representa actualmente es idéntico en todos los aspectos al entorno previamente representado. Pero el tipo apropiado de flexibilidad requiere ser capaz de registrar en qué aspectos hay una coincidencia en el modo en que se representa el entorno y en qué aspectos no hay tal coincidencia, de manera que una respuesta adecuada se pueda determinar

\footnotetext{
${ }^{16}$ La noción de contenido no conceptual se ha propuesto para caracterizar ciertos contenidos mentales que no están constituidos por los conceptos utilizados en su especificación, como el contenido de la experiencia de los niños prelingüísticos, procesos subpersonales y el pensamiento animal (Bermúdez 1995). Gunther (2003) presenta una selección de los textos más representativos del debate en torno a esta noción.
} 
integrando las coincidencias relevantes con la experiencia previa y los estados motivacionales actuales. (Bermúdez 1998, p. 101)

De acuerdo con Bermúdez, a través de la integración cognitiva una criatura puede, por un lado, reconocer lo similar y lo diferente de distintas situaciones, y, por otro, modificar sus creencias y demás actitudes proposicionales en virtud de otras actitudes. Gracias a ello, afirma, una criatura puede comportarse de un modo plástico y flexible, ya que puede aprender de experiencias pasadas.

Ahora bien, la noción de contenido no conceptual no permite explotar la potencia explicativa de la integración cognitiva cuando ésta se encuentra al servicio de la identificación de objetos, esto es, de la identificación de un objeto como el mismo una y otra vez. En sentido estricto, la noción de contenido no conceptual tampoco parece adecuada para explicar los procesos de protoinferencia (Bermúdez 2006), pues éstos también han de requerir alguna comprensión de la identidad. La razón es que para la identificación no basta con agrupar las coincidencias o semejanzas relevantes de una situación. Al contrario, la (co)identificación consiste en nuclear en un mismo concepto un conjunto de representaciones heterogéneas en virtud de referir a una misma sustancia. ${ }^{17}$ De este modo, la discriminación e integración de representaciones sobre rasgos similares y diferentes se potencian con un nuevo uso: el reconocimiento de lo idéntico a partir de lo diferente.

Lo mismo puede extenderse a la noción psicológica de conceptos. De acuerdo con la bibliografía especializada de la psicológica, los conceptos son representaciones almacenadas y recuperadas en la memoria a largo plazo (Weiskopf 2009, Machery 2009). Ahora bien, lo que permite integrar y recuperar la información almacenada en la memoria a largo plazo, para usarla posteriormente, es el hecho de que distintos recuerdos o trozos de información versan sobre un mismo objeto. Por medio de la identificación, la memoria a largo plazo puede funcionar como un sistema consistente y no redundante (Millikan 2000, p. 7).

Así entendida, la identificación constituye un importante logro cognitivo, extendido entre los primates - humanos y no humanosaves y otros mamíferos, pues da lugar a la capacidad de rastrear y

${ }^{17}$ Hay un dejo de ambigüedad en este modo de hablar de la identificación. Para extinguirlo, siguiendo a Millikan (2000), uso "coidentificación" cuando considero la identificación a nivel de los vehículos o representaciones internas, mientras que uso "identificación" para aludir al reconocimiento de la igualdad de contenidos. 
reconocer una misma sustancia $\mathrm{u}$ objeto como la misma cosa aun cuando, en distintas circunstancias, se presente con distintas propiedades y, finalmente, amplía de este modo el repertorio de conductas y pensamientos de la criatura. Este reconocimiento permite, entre otras cosas, comportarse de acuerdo con la información acumulada en experiencias previas de modo que la conducta de una criatura en un momento dado no dependa de los estímulos ambientales inmediatos sino de la información recolectada y almacenada en situaciones anteriores. En consecuencia, la identificación explica por qué se pueden usar de manera eficaz representaciones de rasgos y propiedades que, en un momento dado, se encuentran ausentes o fuera del alcance de la percepción, por qué respondemos de manera determinada incluso en ausencia de los estímulos relevantes o bien por qué inhibimos ciertas respuestas aun cuando los estímulos relevantes se han registrado. $\mathrm{Si}$ bien la noción de integración cognitiva puede formar parte de esta explicación, por sí sola no es suficiente.

Por otra parte, al reconocer que dos grupos de propiedades no son sino del mismo objeto, una criatura puede integrar dos cuerpos de información previamente separados para generar nuevo conocimiento y/o para su posterior uso en nuevas situaciones. Supongamos que una criatura con estados representacionales es, sin embargo, incapaz de vincular diferentes representaciones, $F(a), G(b)$, que tiene de un mismo objeto. En tal caso diremos que si esta criatura descubriera después que ambas representaciones hacen referencia a lo mismo $(a=b)$, habría aprendido algo nuevo acerca de tal objeto, pues ahora sabe que $G(a)$. Este nuevo conocimiento le permitirá exhibir un comportamiento más flexible frente al mismo objeto, pues ahora sabe que éste se manifiesta a través de esas dos propiedades, $F$ y $G$, cada una de las cuales puede formar la base para inferencias, respuestas conductuales y expectativas, todas ellas heterogéneas. Pensemos en una niña que sabe que el gatito mullido recostado sobre el sillón es, además, el furioso animal que clavó las uñas sobre sus manos el día anterior. El comportamiento cauteloso de la niña frente a este animal recostado sobre el sillón habrá de explicarse no en virtud de las propiedades que la niña observa en el animal en ese preciso momento, sino más bien en virtud de que ella lo identifica como el núcleo de ambos conjuntos de propiedades.

En criaturas que poseen conceptos tanto de sustancias como de propiedades, el reconocimiento de que diferentes trozos de información son sobre un mismo objeto permite usar la información representada en inferencias mediatas. Este tipo de inferencia requiere la capacidad de articular pensamientos con una estructura predicativa, 
en la que una propiedad se atribuye a un objeto. Este tipo de inferencia requiere, además, que al menos un elemento se repita y conserve su significado en cada ocurrencia (Millikan 2000, p. 142). Como por ejemplo en: Cicerón era pelado; Tulio = Cicerón; Tulio era pelado. La identificación es, entonces, una precondición para la realización de, al menos, algunas variedades de inferencia mediata, i.e. aquellas que amplían el conocimiento de individuos o clases naturales, por ejemplo. ${ }^{18} \mathrm{Al}$ mismo tiempo, permite explicar uno de los papeles centrales de la realización de inferencias para quien posee conceptos: la ampliación de conocimiento. En cambio, las criaturas que sólo tienen habilidades para identificar disponen únicamente de un conocimiento práctico (know-how) e implícito de las sustancias que, sin embargo, da lugar a un comportamiento guiado y flexible a partir de la información integrada: una criatura que percibe un objeto y lo reconoce como el mismo que vio el día anterior puede integrar la información que recolectó en ambas ocasiones y usarla de un modo novedoso para guiar su conducta. Así, por ejemplo, un animal que no ve un predador pero puede olerlo o sentir su bramido no saldrá de su nido a menos que las señales de peligro desistan.

En síntesis, cuando la integración cognitiva se articula con la comprensión - ya sea implícita o explícita - de identidad de contenidos - es decir, con la coidentificación de representaciones sobre una misma sustancia - y no se reduce, por lo tanto, a la yuxtaposición de propiedades similares y diferentes, amplía el conocimiento y guía la acción. En consecuencia, la habilidad de identificar es imprescindible para explicar los procesos de razonamiento, pues la realización de inferencias presupone alguna comprensión de la identidad y, por otra parte, posibilita un acceso epistémico a los objetos a los cuales hace referencia el contenido del pensamiento. A diferencia de la capacidad de volverse reflexivamente sobre el pensamiento, la identificación se puede entender en términos de un conocimiento implícito $-\mathrm{y}$, en algunos casos, práctico - de las condiciones de "individuación de objetos en el pensamiento". 19

${ }^{18}$ Se podría objetar que el término medio en razonamientos donde sólo hay términos generales no presupone habilidades identificatorias, sino clasificatorias. No obstante, si dentro de la noción de sustancia se incluyen estofas, clases naturales, históricas y sociales, además de individuos, la identificación parece ser un requerimiento también en estos casos.

${ }^{19}$ Es importante tener en cuenta que no sólo hay distintos modos de obtener información acerca de un mismo objeto sino que distintos tipos de conceptos requerirán distintos mecanismos para recabar información y regular sus condiciones de corrección. Por ejemplo, los conceptos de objetos ficticios o históricos tendrán mane- 
Si esto es así, aunque no lo puedo argumentar de manera completa aquí, la identificación también resulta indispensable para dar cuenta del principio de Russell (Evans 1982). De acuerdo con este principio, una criatura debe tener un acceso epistémico al objeto de su pensamiento; en específico, debe tener un conocimiento discriminador acerca de los objetos de sus pensamientos sobre particulares. ${ }^{20}$ Debe saber acerca de qué está pensando, cuál es el contenido de su pensamiento. Para ello, el pensamiento debe estar constituido por elementos referenciales que permitan identificar particulares. Por lo general, se ha asumido que este principio exige un conocimiento proposicional de las condiciones de individuación de los objetos. Ahora bien, la habilidad para identificar sustancias constituye un modo de individuar conceptos para cuya comprensión no es siempre imprescindible un conocimiento explícito y proposicional. ${ }^{21}$ Veamos.

En primer lugar, los estudios en psicología han recolectado evidencia psicológica acerca de la comprensión de la permanencia de objetos, aun en niños en la etapa prelingüística (Baillargeon 1987, Spelke 1990). Ello daría cuenta de que ya existen elementos referenciales en la percepción (Burge 2010). En segundo lugar, la identificación cumple un papel primordial en la adquisición de conceptos, pues justamente se define en términos de la acumulación e integración de información acerca de un mismo objeto (Millikan 2000). La comprensión explícita de las condiciones de individuación de un concepto presupone la posesión del concepto en cuestión, pues la adquisición de conceptos es previa a su evaluación epistémica. Conforme a esta hipótesis, se puede decir que los niños (humanos) comienzan a usar los conceptos e, incluso, les dan una expresión lingüística mucho

ras de recabar información y condiciones de corrección diferentes a la que requieren los conceptos empíricos. En tanto la identificación consiste en la coidentificación de representaciones sobre un mismo objeto, a diferencia de los conceptos empíricos que incluyen la coidentificación de representaciones perceptuales, los conceptos abstractos incluyen, en cambio, la coidentificación de representaciones abstractas o no perceptuales. Pero, para ello, es menester contar con capacidades inferenciales que presupongan una estructura predicativa.

${ }^{20}$ El principio de Russell es un principio que rige los pensamientos sobre particulares y no los pensamientos generales, como "Todos los cisnes son blancos", puesto que ese tipo de pensamiento carece de elementos referenciales.

${ }^{21}$ Bermúdez, por su parte, muestra su adhesión a este principio (2010, p. 46). Sostiene que los objetos del pensamiento de segundo orden —otros pensamientosson individuados inferencialmente; sin embargo, no explica cómo se individúan los objetos de los pensamientos de primer orden. En ciertos lugares, él reconoce que el papel inferencial desempeña sólo una tarea parcial en la individuación de conceptos. Sin embargo, no explicita qué otros aspectos forman parte de las condiciones de individuación de conceptos. 
antes de comprender explícitamente cuáles son sus condiciones de individuación, pues si se acepta que el referente cuenta entre las condiciones de individuación de un concepto, la identificación posibilita una comprensión implícita de tales condiciones.

En tercer lugar, cuando la guían objetivos estrictamente prácticos, la identificación simplemente involucra la capacidad para dar respuestas conductuales confiables y flexibles — aunque falibles - en los diversos encuentros con un mismo objeto y, para esto, sólo se requiere que la criatura sea capaz de integrar la información representada y usarla conforme a sus metas y los encuentros previos con el objeto (Millikan 2000). En este sentido, la criatura tendría un conocimiento práctico o implícito acerca de las condiciones de individuación de los conceptos. Con el fin de lograr este tipo de identificación, no es necesario exigir que la criatura reconozca explícitamente las razones inherentes a la identificación sino simplemente que tenga la sensibilidad práctica para hacerlo. Tampoco se necesita ser capaz de dar justificaciones porque tener un concepto sobre un objeto es saber identificarlo y no ponderar explícitamente las razones que conducen a tal identificación. ${ }^{22}$

Por último, la identificación incluye un importante componente normativo, pues al integrar información sobre una misma sustancia, una criatura se vuelve capaz de modificar y corregir sus conductas, eliminar contradicciones y modificar sus creencias y deseos en la medida en que incorpora nueva información sobre el referente de la identificación y en virtud de su propio éxito y fracaso en encuentros previos con la sustancia, así como en virtud de las relaciones inferenciales entre los pensamientos acerca de la sustancia.

Se puede preguntar, sin embargo, si esta condición es suficiente para el pensamiento conceptual. De hecho, Bermúdez podría conceder el punto y admitir que la identificación de objetos es un importante rasgo de los conceptos, pero insistiría en que la capacidad de identificar sin la de reflexionar no basta para tener conceptos. Supondré, para seguir con el argumento, que no lo es. ${ }^{23}$ Sostendré,

${ }^{22}$ Así como la identificación no implica evaluar la relación lógica entre razones ni involucra juicios de identidad, tampoco implica evaluar "cuán confiables" son los medios empleados para la identificación, como sostendría una posición confiabilista.

${ }^{23}$ Es importante aclarar que, al sostener que la identificación constituye una de las funciones centrales de los conceptos que no requiere pensamiento de segundo orden y que es necesaria para realizar inferencias, sólo he usado la teoría de Millikan (2000) de un modo restringido, pues, a diferencia de Millikan, pienso que la identificación es insuficiente para el pensamiento conceptual. Como argumento a continuación, el pensamiento conceptual involucra una estructura inferencial y predicativa, capaz de 
en cambio, que la generalidad y la identificación de objetos en forma conjunta constituyen dos condiciones centrales para la posesión de conceptos que, por un lado, i) no requieren ascenso intencional, por otro lado, ii) son indispensables para explicar al menos ciertas formas de inferencia, y finalmente iii) ponen al descubierto aspectos significativos del pensamiento conceptual. Lo que quiero mostrar con esto es que Bermúdez nos debe razones por las cuales aceptar que, para ser conceptual, el pensamiento debe ser reflexivo y requiere la capacidad de pensar explícitamente en las relaciones inferenciales entre distintos pensamientos.

\section{b) Generalidad}

El requisito de generalidad ha sido bien aceptado en la bibliografía filosófica sobre conceptos. ${ }^{24}$ En términos generales, según este requisito, el pensamiento conceptual está esencialmente estructurado y se compone de distintas habilidades sistemáticas que son constitutivamente recombinables de modo indefinido en distintos pensamientos.

[C] ualquier pensamiento que podamos interpretar como poseedor del contenido de que a es $F$ entraña el ejercicio de una habilidad —el conocimiento de lo que es que algo sea $F$ - que se puede ejercitar

satisfacer el requisito de generalidad, algo que Bermúdez acepta. Ahora bien, como lo señaló uno de los evaluadores anónimos de este trabajo, se podría preguntar si esta estrategia es legítima. Pienso que ésta se ve posibilitada fundamentalmente por el hecho de que ambas teorías recurren a las nociones de representación mental y de integración cognitiva para dar una explicación de los conceptos y, en general, de la intencionalidad. Por otro lado, pese a sus diferencias, ambas teorías encarnan una concepción realista de los conceptos y consideran que tener conceptos involucra tener habilidades, identificatorias para Millikan e inferenciales para Bermúdez.

${ }^{24}$ Coexisten, sin embargo, diversas acepciones sobre el requisito de generalidad. Las diversas lecturas que ha recibido este requisito, por lo general, difieren en el grado de libertad o permisividad en las combinaciones que se ha de exigir a un sistema para que sea conceptual. Así, por ejemplo, algunos sostienen que si una criatura tiene los conceptos $F$ y $a$, y puede pensar $F(a)$, luego para algunos conceptos $G$ y $b$ que la criatura posea, podrá pensar $G(a)$ y $F(b)$ (Carruthers 2009). Otros, en cambio, consideran que la posesión de conceptos involucra la capacidad de pensar $G(a)$ y $F(b)$ para todo concepto que la criatura posea (Evans 1982, Hurley 2003, Peacocke 1992). Entre estas alternativas, Camp (2009) propone una versión gradualista del requisito de generalidad, que pueden satisfacer criaturas sin lenguaje. Bermúdez, por su parte, ha defendido una lectura fuerte del requisito para defender que los conceptos requieren vehículos lingüísticos. Sin embargo, como señalé al comienzo, en este trabajo dejaré de lado esta cuestión, dado que lo que me interesa no es cuán libremente podemos combinar nuestros conceptos sino el tipo de capacidad que se necesita con el fin de que la combinación sea posible. 
indefinidamente en muchos pensamientos distintos y que se ejercitaría, por ejemplo, en el pensamiento de que $b$ es $F$. De manera similar para el pensamiento de que $a$ es $G$. (Evans 1982, p. 103)

Hay diversas razones en favor del requisito de generalidad. Desde un punto de vista metodológico, provee un criterio relativamente claro para la posesión de conceptos. Pero hay razones más profundas para su aceptación. En primer lugar, la satisfacción del requisito de generalidad garantiza un enriquecimiento significativo del repertorio conceptual de una criatura que tiene habilidades para identificar, pues ésta ha de poder combinar en una estructura predicativa las habilidades para identificar con otro tipo de habilidades representacionales, como las habilidades clasificatorias que involucren, además, conceptos de propiedades.

En segundo lugar, sería extraño hablar de "pensamiento conceptual" si éste no estuviera constituido por partes recombinables, pues la recombinabilidad es lo que garantiza un grado interesante de separación entre una representación y lo representado o, en términos davidsonianos, entre lo objetivo y lo subjetivo, así como una diferenciación entre el pensamiento conceptual y la percepción. Me refiero al hecho de poder "separar" un concepto - por, ejemplo ROJO- de un contenido completo - de la manzana es roja - para que forme parte de otros contenidos — como en las casas son rojas-. Esto depende de la posibilidad de separar la representación — ROJO - de la situación percibida representada — la manzana roja sobre la mesa- y usarla en otros contextos, alejados de esa situación — al recordar, por ejemplo, que había una manzana roja sobre la mesa-. Así, podría decirse que mientras la identificación da lugar a la flexibilidad conductual, la generalidad da lugar a una nueva clase de flexibilidad de tipo cognitivo.

Por otro lado, la recombinabilidad permite explicar la capacidad de utilizar información obtenida en un contexto y aplicarla en otro, como sucede en la realización de inferencias, en la de metáforas, etc. Esta libertad para aplicar nuestros repertorios conceptuales en diversos contextos se puede entender a la luz de la tesis de que los conceptos guardan un conocimiento con cierto grado de abstracción y generalidad respecto de aquello a lo que se refieren. Como en el ejemplo anterior, el concepto de ROJO se puede independizar de la percepción de manzanas rojas y del pensamiento de que las manzanas son rojas.

Por último, el requisito de generalidad exige que los conceptos sean recombinables de modo sistemático, esto es, que realicen la 
misma contribución semántica en los distintos pensamientos de los que formen parte. Sin la sistematicidad sería difícil explicar el razonamiento y la realización de inferencias, puesto que para concluir, por ejemplo, que al menos alguien es feliz a partir de Cata es feliz, necesitamos no sólo que el concepto SER FELIZ pueda formar parte de distintos pensamientos sino también que conserve su significado en cada uno de ellos. En este sentido, es muy convincente que sólo una criatura dotada de habilidades inferenciales pueda satisfacer el requisito de generalidad. Más aún, el hecho de que una criatura sea incapaz de realizar inferencias indicaría que no tiene conceptos. De aquí se desprende que la posesión de conceptos ha de involucrar habilidades tanto para identificar como para hacer inferencias.

Ahora bien, salvo que aceptemos que la capacidad de hacer inferencias se encuentre ligada a la justificación epistémica, este requisito no parece involucrar ascenso intencional alguno. En cambio, implica que un sistema conceptual debe ser productivo y sistemático, de modo que garantice múltiples combinaciones entre sus componentes. Pero, tal como lo han puesto de manifiesto las ciencias cognitivas, estas propiedades se pueden explicar desde un punto de vista causal en términos de reglas sintácticas, ejecutadas por mecanismos y procesos subpersonales (Fodor y Pylyshyn 1988, Camp 2009). En tanto que — según este modelo explicativo - las propiedades semánticas se ven reflejadas en las propiedades sintácticas, para realizar inferencias y combinar conceptos no se necesita un sistema que opere de modo consciente ni reflexivo. ${ }^{25}$

Por otro lado, así como para integrar nueva información con una representación previa de un objeto no se necesita pensar acerca del concepto sino sólo acerca del objeto (por ejemplo, cuando pensamos "Luci es linda además de ser simpática"), del mismo modo, no necesitamos pensar en los conceptos de DEDO ni de PÉTALO (sino en dedos y pétalos) para entender la metáfora "Sus manos se abren como pétalos". Por supuesto que las personas participamos de muchas prácticas reflexivas en las que nuestra preocupación está centrada en los conceptos, en el uso de los conceptos, etc. La teorización filosófica, la explicación científica, la modelización, la revisión de

${ }^{25}$ En esta dirección, Burge (2010) sostiene que para que una criatura razone deductivamente, sus transiciones psicológicas se deben poder explicar conforme a un modelo de razonamiento deductivo. Esto es, sus pensamientos deben ser instancias de esquemas deductivos. La teoría fodoriana da un paso más y asume una perspectiva realista sobre estos modelos, pues supone que el cerebro es un procesador que opera conforme a tales esquemas. Lo interesante de esta teoría es que da una explicación causal de por qué nuestro pensamiento se adecua a ese modelo. 
creencias y deseos, etc., son ejemplos de ello. Lo que quiero señalar es que para combinar conceptos —algo que muchos niños comienzan a hacer desde muy pequeños - no necesitamos pensar explícita y reflexivamente en ellos. ${ }^{26}$

Hasta aquí, he presentado dos condiciones para el pensamiento conceptual. Como he señalado, ninguna de ellas implica ascenso intencional. Sin embargo, si aceptáramos que una criatura satisface ambas condiciones, tendríamos buenas razones para admitir que posee conceptos. No creo haber brindado de este modo una objeción concluyente contra la propuesta de Bermúdez. Sin embargo, espero haber mostrado que la clase de conceptos que satisface el requisito de ascenso intencional excluye buena parte de pensamientos conceptuales.

\section{Observaciones finales}

En este trabajo he intentado esclarecer las motivaciones que llevan a Bermúdez a aceptar la tesis de que para tener conceptos una criatura pensante debe ser capaz de tener pensamientos sobre otros pensamientos. He señalado que el autor apela a una concepción internista de la justificación epistémica. Este compromiso lo hace aceptar, por un lado, que la posesión de conceptos involucra la capacidad de tener una comprensión explícita de las relaciones lógicas y evidenciales entre distintos pensamientos y, por otro lado, que para tener conceptos es necesario ser capaz de dar justificaciones, una práctica paradigmáticamente lingüística.

Contra este conjunto de tesis y compromisos, he señalado las dificultades para dar cuenta del uso de conceptos, la realización de inferencias y la capacidad reflexiva tal como los llevan a cabo los niños y los animales sin lenguaje, con base en un conocimiento implícito de las relaciones inferenciales entre pensamientos. Por su parte, Bermúdez no puede aceptar lo anterior al sostener que este tipo de conocimiento depende de la capacidad de representar explícitamente tales relaciones. Las dificultades señaladas ponen en tela de juicio la tesis de que el pensamiento de segundo orden constituya una condición necesaria para el pensamiento conceptual y, con ello, la tesis de que el lenguaje natural y la evaluación epistémica sean inescindibles de la posesión de conceptos.

\footnotetext{
${ }^{26}$ Mientras Bermúdez diría que el uso habitual de conceptos no involucra ascenso intencional, mi tesis es que la adquisición temprana de conceptos, como el uso habitual, no lo requiere en el sentido de que es el ascenso intencional lo que depende de la posesión de concepto y no a la inversa.
} 
Por último, he propuesto dos condiciones para el pensamiento conceptual que no involucran ascenso intencional y, sin embargo, resaltan aspectos significativos del uso de conceptos: la identificación y la generalidad. He argumentado que la identificación constituye una habilidad indispensable para tener conceptos e incluso para realizar inferencias, mientras que el requisito de generalidad pone en evidencia aspectos distintivos del pensamiento conceptual. De aquí se sigue una concepción mixta de los conceptos, según la cual tener conceptos involucra habilidades tanto inferenciales como identificatorias, sin las cuales no es posible satisfacer el requisito de generalidad.

Tal como las he presentado aquí, estas condiciones requieren un conocimiento implícito de las condiciones de individuación de los conceptos y, particularmente, son independientes de la capacidad de representar explícitamente el pensamiento y las relaciones de inferencia entre sus contenidos. En este sentido, se ajustan a lo que sabemos sobre los niños y algunas especies de animales, según las investigaciones en psicología del desarrollo, etología cognitiva y psicología comparada. $^{27}$

\section{BIBLIOGRAFÍA}

Baillargeon, R., 1987, "Object Permanence in 3.5- and 4.5-Month-OldInfants", Developmental Psychology, vol. 23, pp. 655-664.

Bermúdez, J.L., 2010, "Two Arguments for the Language-Dependence of Conceptual Thought", New Perspectives on Concepts. Grazer Philosophische Studien, vol. 81, pp. 37-54.

—_, 2009, "Mindreading in the Animal Kingdom", en Lurz 2009, pp. 145-164.

— 2006, "Animal Reasoning and Proto-Logic", en Hurley y Nudds 2006, pp. 127-137.

-, 2003, Thinking without Words, Oxford University Press, Oxford.

- 1998, The Paradox of Self Consciousness, The MIT Press, Cambridge, Mass.

${ }^{27}$ Este trabajo se presentó en el I Coloquio sobre Conceptos y Percepción, que se llevó a cabo del 25 al 27 de noviembre de 2010, en Villa General Belgrano, Córdoba, Argentina. Agradezco las observaciones y la discusión de los participantes, especialmente a Laura Danón, Darío Scattolini y Elizabeth Camp. Quisiera agradecer también a José Luis Bermúdez, por su lectura, y muy especialmente a los árbitros anónimos y editores de la revista, por sus comentarios y por alentarme a mejorar el trabajo. 
Bermúdez, J.L., 1995, "Nonconceptual Content: From Perceptual Experience to Subpersonal Computational Status", Mind and Language, vol. 10, no. 4, pp. 333-369.

Boesch, C. y H. Boesch, 1990, "Tool Use and Tool Making in Wild Chimpanzees", Folia Primatologica, vol. 54, pp. 86-99.

Bond, A.B., A.C. Kamil y R.P. Balda, 2003, "Social Complexity and Transitive Inference in Corvids", Animal Behaviour, vol. 65, pp. 479487.

Brandom, R., 2010, "Conceptual Content and Discursive Practice", New Perspectives on Concepts. Grazer Philosophische Studien, vol. 81, pp. $13-35$.

— 2000, La articulación de las razones. Una introducción al inferencialismo, Siglo XXI, Madrid.

Burge, T., 2010, "Steps toward Origins of Propositional Thought", Disputatio, vol. 4, no. 29, pp. 39-67.

Call, J., 2006a, "Descartes' Two Errors: Reason and Reflection in the Great Apes", en Hurley y Nudds 2006, pp. 219-234.

— , 2006b, "Inference by Exclusion in the Great Apes: the Effect of Age and Species", Animal Cognition, vol. 9, pp. 393-403.

Call, J., B. Hare, M. Carpenter y M. Tomasello, 2004, " 'Unwilling' versus 'Unable': Chimpanzees' Understanding of Human Intentional Action", Developmental Science, vol. 7, no. 4, pp. 488-498.

Call, J. y M. Tomasello, 2008, "Does the Chimpanzee Have a Theory of Mind? 30 Years Later", Trends in Cognitive Sciences, vol. 12, no. 5, pp. 187-192.

Camp, E., 2009, "Putting Thoughts to Work. Concepts, Systematicity, and Stimulus-Independence", Philosophy and Phenomenological Research, vol. 78, no. 2, pp. 275-311.

Carruthers, P., 2009, "Invertebrate Concepts Confront the Generality Constraint (and Win)", en Lurz 2009, pp. 89-107.

Cheney, D. y R. Seyfarth, 2007, Baboon Metaphysics: The Evolution of a Social Mind, The University of Chicago Press, Londres.

— 1990, How Monkeys See the World, University of Chicago Press, Chicago.

Clayton, N., N. Emery y A. Dickinson, 2006, "The Rationality of Animal Memory: Complex Caching Strategies of Western Scrub Jays", en Hurley y Nudds 2006, pp. 197-216.

Davidson, D., 1982, "Animales racionales", Subjetivo, intersubjetivo, objetivo, trad. O. Fernández Prat, Cátedra, Madrid, pp. 141-155.

Destéfano, M., 2009, "Una crítica a las nociones de contenido conceptual y no-conceptual de J.L. Bermúdez", en L. Skidelsky, D. Pérez y C. Scotto, Cuestiones mentales, Universidad Nacional de Córdoba, Córdoba, pp. 33-65.

Duhau, L., 2010, "Do Bees Really Have Concepts? A Discussion of Carruthers' Criteria for Conceptuality", Teorema, vol. 29, no. 2, pp. 125134 . 
Eraña, Á., 2009, “La noción de ‘justificación', ¿un concepto dual?”, Diánoia, vol. 54, no. 62, pp. 47-71.

Evans, G., 1982, The Varieties of Reference, Oxford University Press, Oxford.

Fodor, J., 2008, The Language of Thought Revisited, Oxford University Press, Oxford.

Fodor, J. y Z. Pylyshyn, 1988, "Connectionism and Cognitive Architecture", Cognition, vol. 28, nos. 1-2, pp. 3-71.

Glock, H.J., 2009, “Can Animals Act for Reasons?”, Inquiry, vol. 52, no. 3, pp. 232-254.

—_, 2000, "Animal, Thoughts and Concepts", Synthese, vol. 123, no. 1, pp. 35-64.

Gunther, Y.H. (comp.), 2003, Essays on Nonconceptual Content, The MIT Press, Cambridge.

Hare, B., J. Call, B. Agnetta y M. Tomasello, 2000, "Chimpanzees Know What Conspecifics Do and Do Not See", Animal Behaviour, vol. 59, pp. 771-785.

Heck., R.G., 2007, “Are There Different Kinds of Content?", en B.P. McLaughlin y J. Cohen (comps.), Contemporary Debates in Philosophy of Mind, Blackwell, Oxford, pp. 117-138.

Hurley, S.L., 2003, "Animal Action in the Space of Reasons", Mind and Language, vol. 18, no. 3, pp. 231-256.

Hurley, S.L. y M. Nudds (comps.), 2006, Rational Animals?, Oxford University Press, Oxford.

Lurz, R.W., 2007, "In Defense of Wordless Thoughts about Thoughts", Mind and Language, vol. 22, pp. 270-296.

Lurz, R.W. (comp.), 2009, The Philosophy of Animal Minds, Cambridge University Press, Cambridge.

Machery, E., 2009, Doing without Concepts, Oxford University Press, Oxford.

McDowell, J.H., 1994, Mente y mundo, trad. M.Á. Quintana Paz, Sígueme, Salamanca.

Millikan, R., 2000, On Clear and Confused Ideas: An Essay about Substance Concepts, Cambridge University Press, Cambridge.

Mitchell, P., U. Teucher, M. Bennett, F. Ziegler y R. Wyton, 2009, "Do Children Start Out Thinking They Don't Know Their Own Minds?", Mind and Language, vol. 24, no. 3, pp. 328-346.

Peacocke, C., 1992, A Study of Concepts, The MIT Press, Cambridge, Mass.

Proust, J., 2006, "Rationality and Metacognition in Non-Human Animals", en Hurley y Nudds 2006, pp. 247-274.

Spelke, E., 1990, "Principles of Object Perception", Cognitive Science, vol. 14, pp. 29-56.

Strawson, P.F., 1959 (2003), Individuals. An Essay in Descriptive Metaphysics, 2003, Taylor and Francis, Londres. 
Weir, A. y A. Kacelnik, 2006, “A New Caledonian Crow (Corvus moneduloides) Creatively Re-Designs Tools by Bending or Unbending Aluminium Strips", Animal Cognition, vol. 9, no. 4, pp. 317-334.

Weiskopf, D., 2009., "The Plurality of Concepts", Synthese, vol. 169, no. 1, pp. 145-173.

Recibido el 14 de febrero de 2011; revisado el 22 de septiembre de 2012; aceptado el 5 de octubre de 2012. 